\title{
Command and Control System and Fire Control System Overview of an Engineering Protection System
}

\author{
Peichao Zhang ${ }^{1, a}$, Zhi $\mathrm{Yi}^{1, \text { a }}$ Ning $\mathrm{Cai}^{1, \mathrm{~b}}$ and Wenxuan $\mathrm{Xu}^{1 \mathrm{~b}}$ \\ ${ }^{1}$ Engineering Third Research Institute, Luoyang,471023,China \\ a553406497@qq.com, b1196781849@qq.com
}

Keywords: Engineering Protection, Fire Control System, Command and Control System

\begin{abstract}
In the field of engineering protection, ultra-short-range interceptor weapon system is a new protection technology, as a last line of defense air defense network of fire, it is possible for the penetration of precision-guided munitions to intercept important protection to ensure the safety objectives. This paper summarizes the command and control system and fire control system of an engineering protection system, and introduces its definition, composition and working processes.
\end{abstract}

\section{Introduction}

In the military field, engineering protection is through the construction of protective buildings, using a combination of engineering and technical measures to avoid or mitigate weapons on personnel, equipment, supplies or works and other targets of anti-destruction and the implementation of protection [1-2]. With the continuous development of high-tech surveillance equipment and long-range precision strike weapons and a wide range of applications, engineering protection is facing increasing challenges. Traditional "confront enemy soldiers with generals and stem flooding water with earth" the passive protection means no longer meet the operational requirements of protection under the conditions of information warfare, information warfare need to adapt to explore the operational requirements of the new integrated protection technology.

The engineering protection system is a new protection system to intercept short-range ultra-protection technology. The main object is the protection of precision-guided munitions, and it uses precision-guided munitions terminal ballistics relatively stable, to establish the final anti-aircraft fire network, intercepting penetration precision-guided munitions. Command and control system and fire control system is the core component of the protection system of the project, the main task of command and control system is "transmit messages", to generate operational decision support, combat commanders provide services; fire control system is the "brain" of engineering protection system, with a target detection system for solving the shooting moroto parameters, and fire control unit to intercept the target.

\section{Command and Control System.}

Command and Control System refers to an organic whole and combat commander with military affairs management decision support functions such as automatic or semi-automatic equipment, equipment, facilities, according to certain structural relations. Client of command and control system is the commander, and the most important task is to provide decision support for the Operational Command commander. Command and Control System is a variety of weapons and equipment of the "glue" to become operational effectiveness "multiplier" and it plays an irreplaceable role in the operational command [3].

The command and control system of engineering protection system belongs tactical command and control system, in close contact with the weapon system, great emphasis on firepower and coordinated intelligence, real-time decision-making, therefore, while the command and control system is configured independently, but also paying attention to links between it and the other subsystems. Specific connection relationship is shown in Figure 1. 


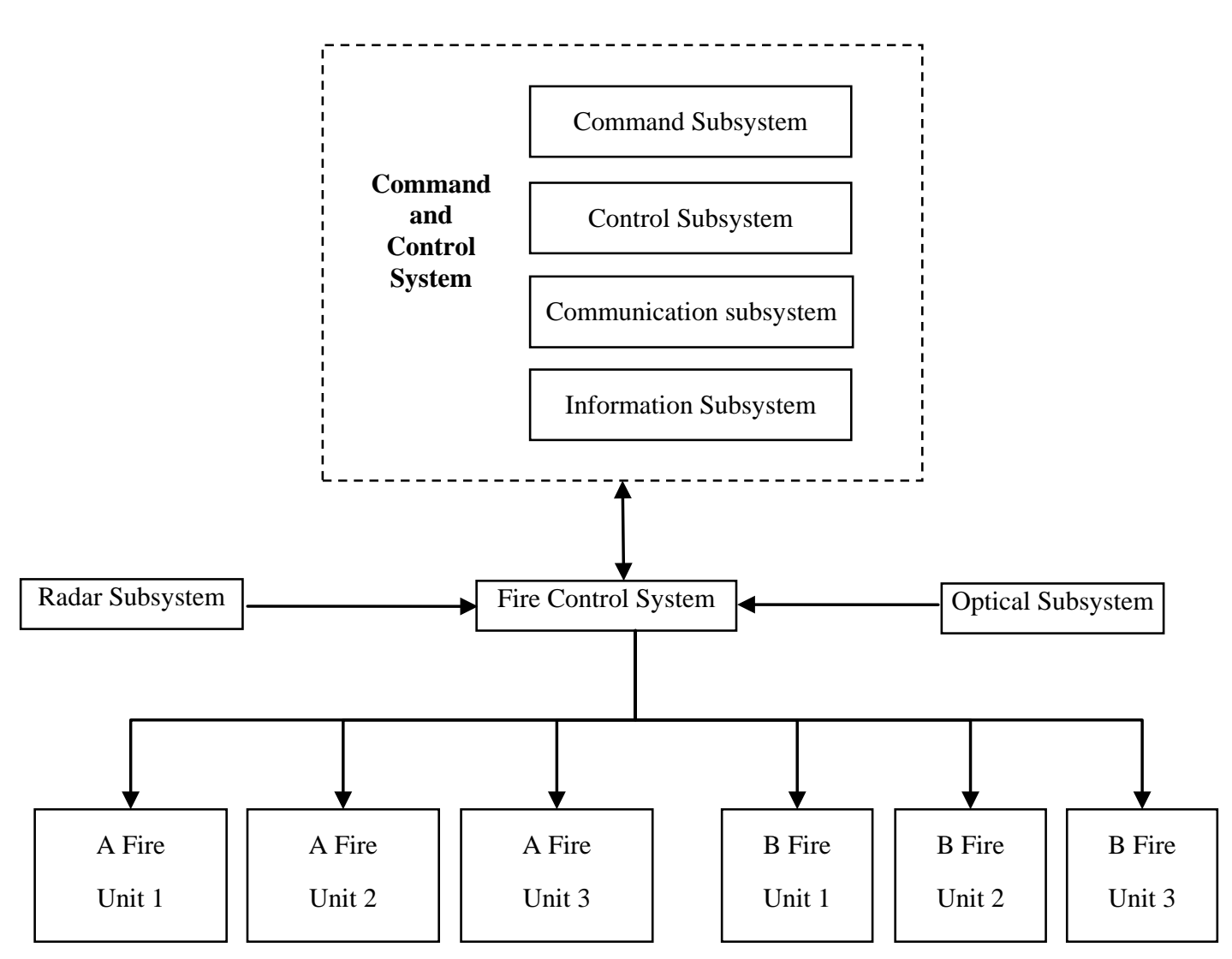

Figure 1 Command and control system and other subsystems connected graph

\section{Fire Control System}

For a complete weapon system, it is usually formed by the fire control system, fire system and delivery system. Among them, the fire control system is an essential weapon system, and is an important part of the weapon system's "brain" and "eyes", and is an important symbol of the advanced nature of the weapon system.

Fire control system is the core weapon systems, and it generally includes target detection systems, fire control computer, ballistic meteorological measurement devices and servo systems [4]. Target detection system for searching, tracking the target, continuous measurement of target coordinates; the main fire control computer to complete the classification and identification of target, threat evaluation, target track fitting and extrapolation, Calculating Firing, interception tactics and fire control unit, interception impact assessment and other tasks; ballistic meteorological measurement means for measuring the ballistic data and meteorological data such as temperature, humidity, barometric pressure, wind speed, wind direction; servo system for weapons adjusted to the direction of shooting. The basic working process of fire control system are: target detection system measures the target coordinates, meteorological measuring device ballistic trajectory, weather and other shooting conditions deviation, the information transmitted in real time computer fire control, fire control computer based on this information and target motion assumed trajectory characteristics of real-time and continuous data required to calculate the firing of weapons, arms by servo system to adjust the weapon firing direction. As can be seen from the above process to work, the main function of the fire control system can be summarized as measurement, calculation and control, which is the core of the fire control computer system.

The engineering protection system is ultra short-range interceptor system, fire control systems and command and control system, target detection system are used in conjunction with the joint, so to a lesser extent included, including fire control computer, fire control switch and meteorological measuring devices. Specific tasks include communication management and information processing, 
situation display, target identification and selection, target track and push the fitting, the shooting parameters moroto calculated interception tactics and fire control unit, blocking effect evaluation.

(1) Fire control computer

By the coordinate calibration and communication, parameters and time synchronization acquisition, target classification and identification, threat evaluation, calculating firing, fire distribution and control, ignition control and security, blocking effect evaluation unit. It used to receive, display real-time status information for each subsystem, complete the trajectory calculated by extrapolation, determine the threat calculations programs and interception calculating firing, guidance and emission ignition and other functions, to achieve command and fire control and interception impact assessment analysis, and can through communication with controllers and other tactical air situation information received Internet connectivity.

(2) Fire Control Switch

48-port gigabit LAN switch, via optical cable with radar, electro-optical tracking device, connected to the fire unit, instrument calibration and other ancillary equipment.

(3) Weather monitoring unit

Get real-time weather monitoring unit for temperature, humidity, barometric pressure, wind speed, wind direction and other information, to provide fire control firing data correction parameter calculation.

(4) Software Module

Including: the main program scheduling module, database management module, fire calculation module (containing the target trajectory fitting module, goals extrapolation module, hit and damage analysis module), visual simulation module, information query module.

\section{Conclusions}

Command and control system and fire control system is an important part of the engineering protection system, the system has a decisive impact on the success of the raid to intercept the target to complete combat missions. For command and control system, as a tactical combat system, real-time command and environmental adaptability of its decision-making is very important, it is the focus of future research; for the fire control system, whose main task is to control the weapon to the target intercept, therefore, target information measure, firing and other count accuracy and timeliness, it is the focus of future research.

\section{References}

[1] Daming Zhu. Discussion on "Engineering protection" concept [J] Protection Engineering, 2011, 33 (5): $72-78$.

[2] "Encyclopedia of Military Engineering" Editorial Board. Military Engineering Encyclopedia [M]. Beijing: Weapon Industry Press, 2012: 91-93.

[3] Yuanzeng Cheng etc. Introduction to fire control system [M] Shijiazhuang: Ordnance Engineering College, 2007: 8-10.

[4] Shiyu Sun etc. antiaircraft fire control system theory [M] Shijiazhuang: Ordnance Engineering College, 1998: 1-2. 